in a ward. The washing of the staff was sent out, as the laundry was incomplete, and there never has been proper provision to wash the linen of the staff. Some of the staff slept outside. . .... Cases of small-pox have occurred owing to these wants of precaution in the families of members of the staff and their friends year after year. For four years no sort of repairs, limewashing, painting, \&c., of the buildings took place. . . . . . The clothing of a patient has never been washed on his admission. The non-resident staff are obliged to work in the wards and among patients in their shirt sleeves, which part of the dress is never changed. .... Cases of small-pox have occurred among members of the committee and their households."

Mr. Power, in his report, has most carefully endeavoured to assess the infection value of some seventy "occasions day by day for communication between the hospital and the district around it" during the infection days Jan. 12th to 17 th of "his outburst" in 1881-or a total incoming and outgoing of 439 , including the arrival and departure of eighteen ambulances, - but of this we would remark, in the words of the Royal Commission (p. xxv.): "It is very difficult to estimate the value of $\mathrm{Mr}$. Power's analysis. .... We will only say that it is very careful, but that it must be accepted subject to all that we have said respecting the extent to which small-pox is capable of being propagated through unexpected and undiscernible channels."

(To be continued.)

\section{AN OPERATION FOR HALLUX VALGUS.}

BY ARTHUR E. BARKER, F.R.C.S. ENG.,

ASISTANT PROFESSOR OF CLINICAL SURGERY AT UNIVERSITY COLLEGE AND ASSISTANT-SURGEON TO UNIVERSITY COLLEGE HOSPITAL, ETC.

THE deformity hallux valgus is one for which it will probably be admitted a cutting operation is rarely demanded. In the first place the condition being one due in almost every case to ill-fitting boots, which force the great toe out of the line of its metatarsal bone into strong abduction, it is possible by proper attention to the shoeing of the patient, by methodical manipulation of the faulty toe, and by placing pads between the latter and its neighbours to bring it in most cases into a fairly comfortable position once more. Still every surgeon must meet from time to time with cases of this deformity which baffle all ordinary endeavours to bring about even a tolerable state of things, and where the great toe lying crosswise, either under or over its fellow, gives rise to such inconvenience, if not actual suffering, as practically to cripple the individual so affected. Under these circumstances patients are willing to undergo any treatment in order to be relieved, and will even urge the surgeon to amputate the offending member, which is interfering with all comfort in progression.

The following case will illustrate remarkably well the evils of this condition, and will serve also for the demonstration of a simple operation which was practised with perfect success for its cure.

$X$ - , aged nineteen, was brought to me as an outpatient in November, 1882. He complained much of being crippled by the deformity of his toes. I found both feet to present the deformity in question acutely, but most marked on the right side, where the nail of the great toe lay under its fellow, its matrix being in a state of suppuration from the pressure and irritation, and the parts exquisitely teuder. Various measures had been tried before I saw the patient to relieve him, attention being particularly directed to the in flamed matrix and ingrown nail. 1 did not anticipate much difficulty in relieving the patient, and treated him by cutting out the embedded portion of nail and dressing the ulcerating surfaces with antiseptic materials placed thickly between the toes. This, however, proved of no permanent benefit to the patient, for on trying to walk the great toe always reassumed its vicious position under that next it and the ulceration commenced as before. Finally, after a long treatment the patient's father and a friend came to me and urged me to take stron cer measures, and if necessary remove the toe, as the youth had been thrown out of work and was unable to take any exercise for a long time. I was of course averse to amputation of the member, though it was only an encumbrance, and began

26 British Medical Journal, Oct. 21st, 1882. to review for the students present all the measures which have from time to time been resorted to for improving the deformity. In doing so I was dwelling on the question of excision of the metatarsal phalangeal joint recommended by some, when one of our students, Mr. C. Hoar, asked me if the condition was ever relieved by osteotomy of the metatarsal bone and straightening of the toe thus without interfering with the joint at all, as he had often seen me operate for genu valgum. I told him that I did not think it had been cone hitherto, but that the idea seemed such a good one that we should put it in practice in this case at once. The patients and his father accepting the proposal to do so with great readiness, I performed the following simple operation with the strictest Listerian antiseptic precautions. Having first made an incision through the soft parts about an inch long, on the inner aspect of the metatarsal bone, above its head, I cut out with a chisel a small wedge-shaped piece of bone from the shaft just above the head, the base of the wedge being on the inner surface of the bone, and the apex at a point nearly through to the other side. Then, by forcibly adducting the great toe, I snapped the uncat remainder of the metatarsal bone, and brought the whole member into a straight line. In this position it was easily retained by a narrow splint along the inner border of the foot. The wound was dressed antiseptically, and the patient was allowed to go home at once. But little remains to be said about the case. The wound was left undisturbed for ten. days, and was then found practically healed and without suppuration. The splint was, however, left on for a few days longer, after which, as the bone had united in its now straight position, the patient was allowed to return to work, which he was soon engaged in as actively as ever. I have seen him several times since at intervals (yesterday, Feb. 18th, 1884, for the last time-i. e., over two years after operation), and the toe is almost quite straight, although a little bent by a bad-fitting boot since worn. He is quite relieved of all the troubles incidental to this deformity.

It will be observed from the above short description that this simple operation is almost the same as that we are in the habit of performing for genu valgum. Here, however I thought it better to remove a small wedge of bone instead of simply dividing the part transversely, as in the case of the knee. I imagined there might otherwise be some difficulty in bringing the parts into position, remembering the short leverage of the toe as compared with that of the tibia. The course of the healing process was as completely satisfactory as these osteotomies usually are under the antiseptic treatment of wounds. It was without suppuration, pain or fever. The toe has been a little stiff since, as might be expected, but the patient does not wish any forcible flexion of it, as the stiffness gives him no inconvenience.

I have purposely avoided going into minute details of this operation. Its principles and practice must be perfectly clear to anyone familiar with osteotomy for genu valgum now so commonly practised. It is quite simple and free from risk if performed with strict antiseptic precautions, and though not often called for, still I cannot help thinking that there is a useful place for it in surgery.

This little operation may have been done before, but I cannot find any record of such a procedure. If it be original in this case, the merit of the suggestion lies with one of our students at University College Hospital.

Harley-street, Carendish-square, W.

\section{SOME COMMENTS ON}

\section{LETHARGIC STUPOR OR TRANCE}

\section{AS ILLUSTRATED IN THE CASE RECENTLY REPORTED.}

$$
\text { By A. M. BROWN, M.D. St. AND., \&c. }
$$

The proposal to increase the means of studying diseases of the mind and nervous system in a less exclusive sense than at present exists is certainly a wise one. What stronger argument could be advanced than the late case of lethargic stupor or trance so fully reported by Dr. Gairdner of the Glasgow University in TILE LANCET of Jan. 5th and 12th and Feb, 22ad? The reticence of medical circles regarding this instance is instructively surprising. What can be the reason? Can there, after all, be something considered "no canoy" about such coaditions? Or is it that in England we 
are still unacquainted with the nature of such ailments? Science does not admit of the first, and assuredly the means at our command do not justify the second. The literature of the subject is abundant, and if the home field of study does not suffice it can easily be supplemented elsewhere. A few months' association with the libraries and clinical courses of Salpetrière would go far to supply any want the profession may feel. Perhaps for the moment we are too much engaged in mimicking Cuntinental germ pathologists and physiological experimentalists to find time for noting the minor maladies of the brain and nervous system; the scientific medicine of the day apparently requires the study of phenomena more tangible and material. If a case of lethargic stupor or trance could afford scope for microscopy or micro-germ research, no doubt the interest would be greater. A malady without a microzyme may be a little commonplace, and ill requite the culture craft in vogue; but though it supplies no space for antiseptic measures, disorders of this class are nevertheless realities, and merit the attention of the medically scientific. The case of Mrs. McI —, so fully reported by Dr. Gairdner, is clear enough, and leaves little room for doubt or difficulty. The poor woman, recently confined, low and anæmic in condition, with accompanying mental depression, becomes moody, loses her appetite, and finally refuses food altogether. The trance state sets in suddenly, not fully delined, however, as for several weeks there are certain actions in compliance with the calls of nature, unquestionably, if unconsciously performed, and naturally these are the last to disappear. This presents us with a phase of instinct not always free from danger, and which operates the more powerfully the lower we descend in the social scale; longcontinued culture is required to uproot it, even in those of cruder natures, and where we should least expect to find it. But even this passes as the instinctive and reflex sensibility decreases, and finally she falls into a state of almost absolute unconsciousness and all but perfect anæsthesia, which continues for more than three months, and which is for the first time broken by a visit from her husband, from whom she had all that time been separated by her removal to hospital. Though still unconscious, it is then the patient becomes sensible of a presence, of a touch, of the accents of her native tongue; her eyes become suffused with tears, and the first articulate attempts are Gaelic words of prayer. From this moment she shows signs of returning sensibility, and when her husband repeats the visit on the following day she sits up in bed, and with some affectional emotion she asks about her children and her home, as if she had been absent only a few days.

Such is the simple history of this particular case; and in it, so far as $I$ can see, there is nothing but what has occurred before in the history of human ailments, and will occur again from time to time, as all who are acquainted with the subject of this state must feel perfectly satisfied.

For those acquainted with the subject of vitality under the abnormal aspects which this case presented, a sufficiently clear conception of its nature may be easily gleaned. The simple outline, without commentary, of the conditions personal and associated of the patient, leave little to desire. There is the ordinary example of the wife and mother of the little imaginative humble Western Highland classes, recently confined, low in health and nervous tone, but showing total absence of the physical and moral phenomena which go to constitute the mystically favoured of religiously fervid Catholic circles, or the hystero-cataleptic and hystero-epileptic of a Salpétrière clinique. For those familiar with the various types of either class and the multitudinous literature relating to them, Dr. Gairdner's report should enable them with ease to differentiate the relative position of this particular case in the category of nervous functional derangements to which it evidently belongs. Those cases referred to by Dr. Gairdner as coming more or less under the designation of lethargic stupor or trance, and which he speaks of as either having seen himself, or finds reported by others, are of a very mixed character, and present even in the Carlisle case, in which he finds identity, no very clear resemblance to the one in question. There are very marked pathological conditions distinguishing the former as different even in kind. The tendency to intermittent muttering and low delirium, as in typhus fever, and the unconsciousneas being by no means either so deep or so persistent, and the return of sensibility and desire for food but gradual, show the affinity, both clinically and pathologically, to be remote. Naturally we must not expect to find such a portrait as that which Dr. Gairdner's case supplies in the pathological clinique of the Salpétrière; its exhibits are of quite another order, if some times show. ing some resemblance. Their widely different and more strongly pronounced pathological conditions explain the reason of their being there, as also the necessity of arbitrary modes of feeding common to all establishments for the cure of the insane. In this clinical school of mental and nervous maladies a familiarity with the nervous types is an easy matter. It is when we come in contact with the various forms of "anæstheso-neurasthenia" (if I may be allowed the term), with their exceptionally inten. sified, disordered sentient phenomena, periodic or continued, that the great difficulty begins. It is here that we fail to detect or grasp the conditional pathological processes pre. sumably related to the anomalous manifestations of organic and animal life, in which the whole systemic functional orders, assimilative, nutritive, sensory, and mental, exbibit departures from those normal types which in our present state of physiological knowledge leave us ignorant of every. thing save their visible phenomena, while awaiting the weight of accumulating facts of observation to throw further lighr upon the subject. Ecstasy, catalepsy, and trance, in their various forms and intensities, from simple lethargic stupor to long-continued and complete insensibility, conditions apparently consistent with organic integrity, must be provisionally regarded as pathological expressions whose mystery is evidently confined to neural dynamical perversion, central and peripheral, and sometimes so purely so that it may easily be induced voluntarily from within or arbitrarily from without. For demonstrations of these ecstatic and hypnotic states, it is not necessary either to visit the contem. platives of Mount Athos or to witness the mesmeric enter. tainments of the London Hall of Science.

Ecstasy, catalepsy, and trance, in their various modifica. tions and intensities, constitute a pathological group possessing markedly a typical significance which would render them easily distinguishable if they more frequently presented themselves. Seldom met with in our Protestant and less emotional communities, they are liable to be interpreted through the medium of that ill-digested and heterogeneous literature which, in a condensed form, makes up the Medical Dictionary articles on the subject, productions of invariable traditional colouring, in which the rarest and most mar. vellons instances occupy the prominent positions. When an isolated case of this category occurs, such as the one in ques. tion, a clear and impartial scientific estimate of it is not easily obtainable. The diagnosis, analysis, and treatment are always indirectly influenced by public curiosity or deference to conventional notions of a sentimental or supernatural kind, more troublesome than convenient, even when worthy of respect. The scientific positively disposed are apt to yield too much to prevailing prejudices in dealing with the facts of exceptional nervous morbid forms which come before them. Quite recently the Warlomont fiasco in the Belgium Academy of Medicine, referred to in the report, supplied an example in illustration of this fact. M. Warlo. mont, the learned perpetual secretary, in summing up the value of the various ecstatic, cataleptic, and trance conditions of the girl Louisa Lateau, and speaking of her perpetual fastings, visions, and representations of the Messianic passion with its agony details wrought out in the mystic hæmorrhagic stigmatisms, thought to avoid offending the metaphysical St. Peters on the one hand, and the positive St. Thomases on the other, in complacently withholding a definite opinion as to their nature, prudently preferring to leave the marvellous morbid eccentricities to the inscrutable operation of le diable ou le bon Dieu. But this time-serving eclecticism proved a fall for the honourable academician, from which he is not likely to recover, in the eyes of many. The fideles might console themselves with St. Augustine, "Credo quid absurdum !"-as for the scientific there was nothing felt but shame for the position, and they could only mutter, as MM. Boens and CharbonnierDebatty did audibly, "Autrefois l'on bâtissait le surnaturel sur l'ignorance, aujourd'hui on l'édifie sur le mensonge." Doubtless such influences as I have referred to are difficult to avoid entirely, but they show how necessary it is for those engaged in the study of natural science to remember that, for the truly scientific mind, questions of materialism or immaterialism find no place; it deals simply with phenomena and the necessary conditions of their manifestations, the rest is left to metaphysics and the world of idealities.

The case of Mrs. McI —, as reported, presents us with 
an instance unique in its simplicity. It is scarcely possible to conceive of one with more absence of actual disease or mental complication. Though coming on suddenly, it is so gradual in its intensity development, that there remains for weeks the apparent spontaneity in connexion with what has been called the reflex function of the brain, so much so as to "enable the patient to obey the important calls of nature in a quasi-automatic manner, and to be moved to do so while alone rather than in the presence of others"-a mode of action which in a conscious state is very natural. Moral habits often become instinctively imperative, and the fact of this one persisting under such circumstances can only be "perplexing" when we persist in making functional determinism square with preconceived notions based on a normal state of the organism. Dr. Graham is probably not far wrong when he states in his report that "it was his conviction that if she had been as closely watched at home as she was in the infirmary, she would have passed her evacuations in bed, rather than have allowed herself to be seen moving a limb." In a strictly scientific spirit, we must regret such a choice was not permitted her. It says much for the poor patient's innate sense of propriety under social conditions where it is generally supposed the least likely to be taught.

In the presence of such facts and phenomena as these, though we may have no explanation to offer, it is surely our duty as unobtrusively as possible to patiently observe and not disturb or trifle with them until we know decidedly their meaning and significance. The same applies to nourishing under similar circumstances. In this particular instance the stupor had not reached its full intensity till some time after the forced feeding was begun, and that state continued for months in spite of it. Much is made of this phase of the treatment and the mode in which it was carried out. The speculations with regard to the advantages scientifically gained are liable to objection, and may legitimately be questioned. If it may be reasonably assumed that feeding saved the life of the patient under the conditions, it may as reasonably be assumed that not only was this not the case, but that the condition (trance) was probably prolonged. The probabilities are (judging from analogy with other similar instances), if this case had not been so treated, its duration might have been much shorter, leaving little but debility to remedy, and I have no hesitation in thinking so. It was not artificial measures which aroused the patient into consciousness-the influence of the husband's presence is there in evidence (very probably the "peat reek" smell played no mean part in consummating the event)-and had she not been fed at all, and had remained much longer in the state of insensibility, the case would not have been so very remarkable when compared with many others of its class. It may not be quite explicable why, under certain circumstances, or under any circumstances whatever, a human being can vegetate for months or for years without solid food, but many have done so, and others will do so again; and what is more, under conditions in which feeding would be absolutely fatal. The necessary amount of systemic organic force consistent with that variable state called vitality, even with conscious life, seems over-estimated. So familiar are we with the normal types of nervous derangements, that we refuse to see varieties beyond very narrow limits, and claim to ourselves a capacity for analysing their phenomena far beyond our powers of even noting or detecting them. Feeding trance is very much like feeding fevers, a method very liable to abuse, and which no amount of experience or intelligence can enable $u s$ to adjust in a positive sense. The organism when profoundly absorbed in those conditions relaxes its allegiance to surrounding media and seems independent of the outer world. Too busily engaged in utilising the structural stores of nutritive material at command to inquire or tolerate with impunity, except within given limits that we would impose on its perverted forces, it reminds us of organic laws, and the duty of endeavouring to read aright their just significance before venturing to combat them, no matter on what principles or with what intention; a duty all the more incumbent as such states so rarely come before us. In this singular corner of the physiological domain an order of things prevails altogether novel, and seemingly inverted, and which sets at defiance formal economic theories supposed as universal. Principles of supply and demand, consumption and production, as applicable to the human body even in disease, as to the body politic, may here sometimes seem to be excluded. In clear accounts we know the expenses and receipts must balance, but the operations in such cases are too abstruse for our manipulation. Still, the organic laws of nature which regulate the proportional relations between digestion, assimilation, and excretion must obtain, though outwitting our detection, and their apparently absolute abeyance need not necessarily entail danger, either to life or hopelessness of ultimate recovery. In this respect man does not differ in kind but only in degree from other organised creatures lower in the scale of being. The marvellous vitality capacity which he in common shares wilh them, even in the normal health conditions, when limited to air and water only as a source of nourishment (facts familiar to us all), ought to supply some solid grounds for working out the physiological problems which fasting in the state of trance involves.

To this form of systemic adaptability, so mysteriously magnified by more or less gradual preparation through obscure and elementary morbid processes, it is surely presumption to assign any definite limits. Dr. Gairdner has clearly expressed this view, when, in speaking of the category to which the case which he reports belongs, he remarks that "one is struck by the almost uncontrollable disposition to regard such cases as altogether outside the boundaries of true physiological science ;" and, in continuation, observes, "it is certainly under the head of the disorders" mentioned-ecstasy, catalepsy, trance, and the like, "not to those epileptic forms in their well-recogaised clinical types, that the supernatural origin of the phenomena, whether entertained or not, must fall to be argued." Here we have those pathological distinctions with their respective claims which we have been insisting on throughout clearly admitted, and one cannot help expressing surprise that in Mrs. McI 's case there was done, or allowed to be done, so much more than, judging from the nature of her condition, was apparently necessary. As for psychological phenomena, which in any case are but a part and not the whole, fortunately they do not complicate the subject. There must be something to which the trance state only appears as incidental to admit of such an association. When this occurs, however, it cannot fail to be misread by some, and the reporter has done well in reminding his readers that the time has obviously not yet gone by for the possibility of the discussion of their "morbid supernatural origin." Notwithstanding all the culture and scientific instincts of the age (as Dr. Gairdner finally puts it) or of the age to come, there is little likelihood of its being otherwise so long as humanity in the mass remains constituted as it is. Scientifically, however, miraculous etiology is inadmissible, and as for the inauguration of "the reign of law," its reign being already demonstratively acknowledged as universal and eternal, man may henceforth save himself the trouble of attempting it. To escape the embarrassment and inconvenience of these facts, those in quest of positive truth will do well to accept them as their principle and guide. In the teaching of the natural sciences, "law in nature" forms the only text we have to deal with, and our duty is to study and to learn it in all its range and operations in so far as our finite faculties enable us.

In concluding these comments, I may be permitted to add that if death from inanition during the state of trance, simple and unassociated with insanity or paralysis or any appreciable organic disease, is a possibility which has yet to be observed and recorded, the same applies to being buried alive. Under conditions of marked indications of vitality, such as bodily warmth and respiration-in fine, all save consciousness and sensibility-such an accident would seem impossible, as was remarked on reference to the case under consideration.

Keppel-street, W.C.

\section{THE TREATMENT OF UTERINE DISPLACE- MENTS BY MEANS OF MEDICATED TAMPONS. ${ }^{1}$}

BY ROBERT BELL, M.D., PHYSICIAN TO THE GLASGOW INSTITUTION FOR DISEASES OF WOMEN.

ALTHOUGH versions and flexions of the womb may in a few exceptional cases exist without producing very urgent symptoms, and though these displacements do not endanger life, they as a rule cause so much discomfort and give rise to such a variety of reflex symptoms and painful sequelæ,

I Read before the Edinburgh Obstetrical Society, Feb, 13th. 